\title{
Experimental investigations on the effectiveness of electromagnetic actuator as sensor
}

\author{
Benjamin Defoy, Laurence Genty and Jarir Mahfoud ${ }^{a}$ \\ Université de Lyon, INSA-Lyon, LaMCoS UMR 5259, Villeurbanne, France
}

Received 12 February 2013, Accepted 24 June 2013

\begin{abstract}
The study aims at examining the behaviour of electromagnetic actuator as inductive sensor. The electromagnets are integrated in an alternative Wheatstone bridge and powered with alternative tension. This circuit allows the measurement of the electromagnet inductance variation, and as this variation is a function of the air gap, the displacement can be deduced. The effect of eddy currents is assessed in order to choose the suitable bridge frequency. Finally, a demodulator is used to obtain the tension proportional to the air gap value. The behaviour is assessed experimentally. Experiments are carried out on a simple beam; clamped at one end and simply supported at the other. The displacements could be measured by using four eddy current sensors and the inductive sensor. The inductive sensor displacement and a modal approximation of the four eddy current sensors resulting from impact testing are compared. Analysis of the results obtained predicts an efficient and robust behaviour.
\end{abstract}

Key words: Self-sensing / sensors / electromagnetic actuator / vibrations / experiments

\section{Introduction}

Active Magnetic Bearings (AMB) are nowadays successfully utilized in several industrial applications $[1,2]$. Their main advantages are the contact-less working environment, no sealing constraints, the frictionless suspension, and that they represent an active system. A typical active magnetic bearing system consists of an actuator, a displacement sensor and a digital controller.

The actuators can only produce attractive electromagnetic force and have strongly nonlinear characteristics due to the fact that this force is current and armature position dependent [3]. Usually, and due to technological constraints, the sensor is placed beside the actuator (as close as possible) and the armature position is estimated from the measurements stemming from the sensor. This estimation is acceptable in the presence of rigid body modes but could lead to undesirable effect when crossing flexible mode zones. On the other hand, and as a consequence of the AMB' success, we are looking for reducing its size such that it could be used in small structure like automotive turbocharger.

AMBs (or actuators) which estimate the rotor position from information of the electromagnet signals are referred to as self-sensing actuator. To our current knowledge, the notion of self-sensing AMBs was first introduced

\footnotetext{
a Corresponding author:

jarir.mahfoud@insa-lyon.fr
}

by Vischer and Bleuler [4]. This work examined the problem of control of a single axis AMB with an opposed pair of magnets. Self-sensing magnetic bearings have been investigated by various researchers. Their efforts can be grouped into two main categories. One category is parameter estimation where the bearing air gap is treated as a time-varying parameter of an isolated dynamic magnetic system and amplifier combination. The other approach is to treat the magnetic bearings and the supported object as a whole. In this case, the position of the supported object is a state rather than a parameter [5-9]. Self-sensing magnetic bearings are not only a research concept but now find commercial application [10].

Generally, inductive sensors have the same architecture as electromagnetic actuators, i.e. two copper coils winded around two iron magnetic cores. Nevertheless, the two devices have not the same specifications [11]. The actuators are designed usually for low frequency range (up to $2 \mathrm{kHz}$ ) with high force capabilities. And, the sensors are designed for high frequency range $(10 \mathrm{kHz}-50 \mathrm{kHz})$. Consequently, sensors are made of small magnetic circuit in order to avoid losses into their iron core and actuators have large coil in order to obtain high magnetic field. The two objectives are opposite since the coil size defined the iron core length [3].

This work is a contribution aiming at the experimental assessment of an ElectroMagnetic Actuator (EMA) behaving as inductive sensor. Only one action line 
is considered, and the electronic device is completely changed to insert the electromagnets in an alternative Wheatstone bridge. Electromagnets are powered with an alternative tension. This circuit allows the measurement of the electromagnet inductance. As the inductance is a function of the air gap, the displacement can be deduced. The effect of eddy currents is assessed to choose the bridge frequency. Finally, a demodulator is used to obtain a tension proportional to the air gap displacement value.

Another objective is to assess the possibility of estimating the displacement at the actuator position (colocalized displacement) by using modal approximation. Usually, the colocalized displacement could be rebuilt by using state estimators $[8,9]$, in this study we choose to rebuilt this displacement by using modal interpolation of the measurement issued from the (non-colocalized) eddy current sensors utilized. This approach has the advantage to be simple, easy to implement and less time consuming. This type of estimation was used in a previous study where the system was controlled successfully [12]. The displacements are measured by using four proximity sensors, located along the beam axis. Their positions are chosen as a function of the mode shapes present within the utilization frequency range. The measurements, issued form the Inductive Sensor (IS), are compared to the value resulting from the modal estimation.

After this introduction, the experimental setup is presented then; the principle of the IS will be detailed. In order to illustrate the principle of the modal approximation, a preliminary numerical investigation was performed and discussed. Finally, the experimental results are presented and commented and the conclusions are dressed.

\section{Experimental setup}

The experimental apparatus is composed of a steel beam with constant rectangular section. The beam is clamped at one end and simply supported by a 17000 N.m ${ }^{-1}$ stiffness cylindrical spring at the other end. The actuators are placed $0.368 \mathrm{~m}$ from the clamped end. This position is chosen as a function of the mode shape so that the measurements stemming from the actuators can be assessed with the influence of the first four modes.

The displacements are measured by using four proximity sensors (Vibrometer TQ 102) located along the $y$ axis, namely, $\mathrm{C} 1$ to $\mathrm{C} 4$ (Fig. 1). The real time data acquisition and signal processing is performed by using the A/D-D/A and DSP Dspace ${ }^{\circledR}$ specialized cards. The four first modes of vibration of the beam are considered in this study. Thus the sampling frequency was set to $4 \mathrm{kHz}$. This enables a good description of four modes. In addition, the sampling frequency was high enough to avoid using anti-aliasing filters for the frequency range studied. An impact hammer Brüel and Kjaer (B\&K) 8202 supplied with a piezoelectric force sensor ( $\mathrm{B} \& \mathrm{~K} 8200$ ) is used in order to generate the system responses to the different excitations applied.

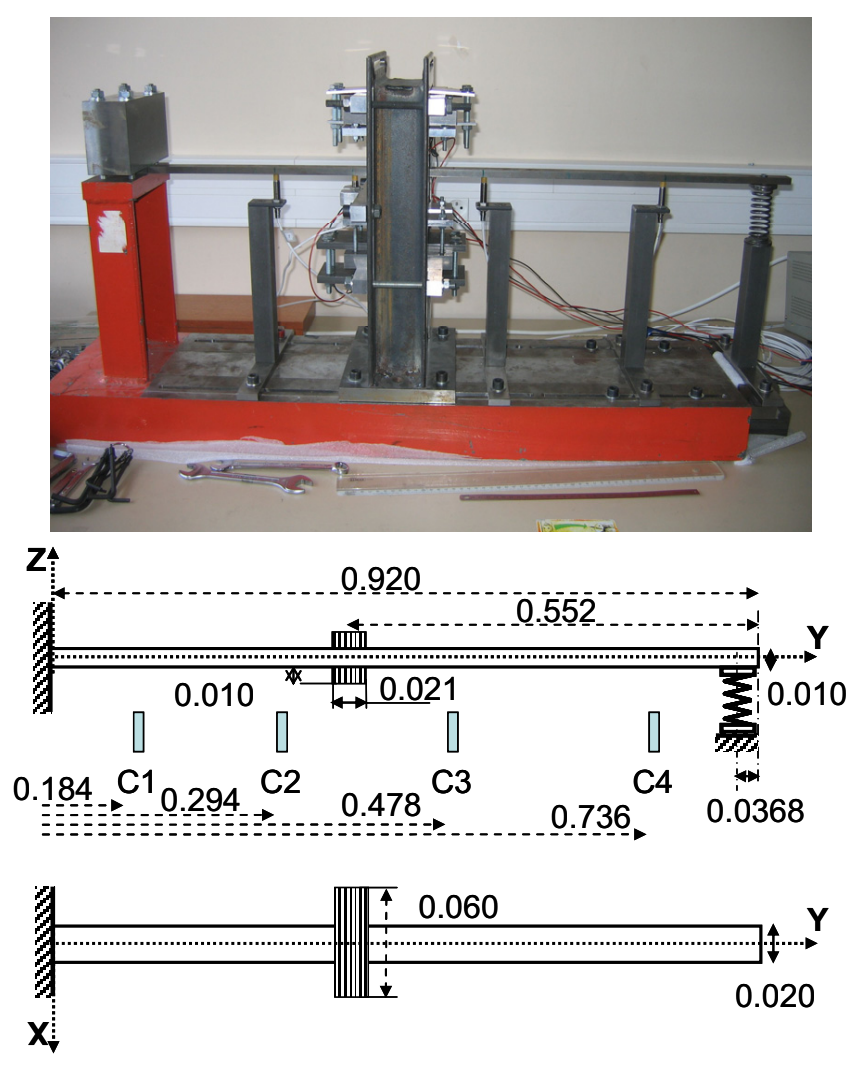

Fig. 1. Experimental setup.

\section{Description and principle of the sensor}

Since an electromagnetic actuator (EMA) can only produce attractive forces, two identical actuators commanded independently are necessary. Each EMA is composed of a ferromagnetic circuit and an electrical circuit. The ferromagnetic circuit is composed of two parts; an $(E)$ shape that receives the induction cooper coil and an (I) shape part that is fixed on the beam. Both parts are composed of assemblies of insulated ferromagnetic sheets. The quality of the ferromagnetic circuit alloy is considered high enough and the nominal air gap between the stator and the beam is small enough to consider magnetic loss as negligible. This EMA was designed and assessed in a previous study [13], it produces a maximum force of $300 \mathrm{~N}$ for a maximum current of $3.5 \mathrm{~A}$ (Fig. 2).

Only one action line is considered, and the electronic device is completely changed to insert the electromagnets in an alternative Wheatstone bridge (Fig. 3). Electromagnets are powered with an alternative tension. This circuit allows the measurement of the electromagnet inductance:

$$
L=2 a f \frac{\mu_{0} N^{2}}{2 \delta_{\mathrm{a}}+\frac{l}{\mu_{\mathrm{r}}}}
$$

where:

$\mu_{0}=4 \pi \times 10^{-7} \mathrm{H} \cdot \mathrm{m}^{-1}$ the permeability of vacuum space, $a=$ sensor geometry (Fig. 2),

$f=$ sensor geometry (Fig. 2),

$N$ is the number of turn in coils, 

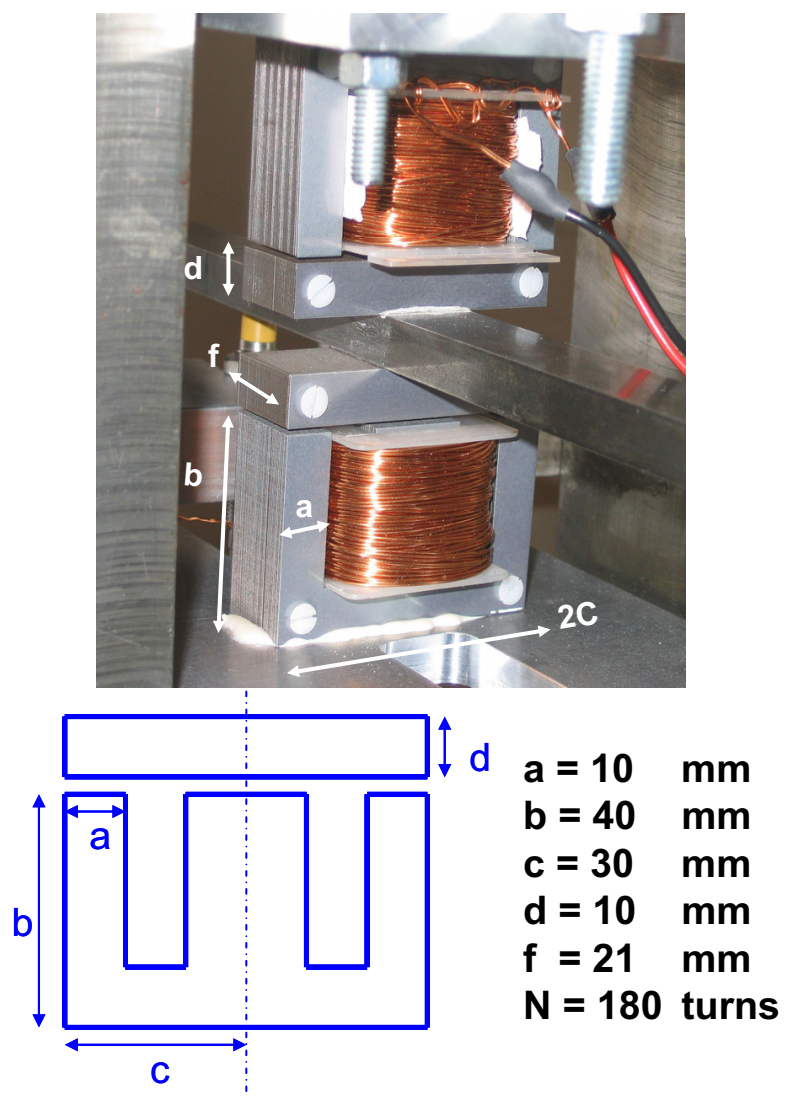

Fig. 2. Sensor details.

$\delta_{\mathrm{a}}$ is the colocalized displacement $=e_{0} \pm x$,

$e_{0}$ is the initial air gap (the distance between the $I$ and $E$ shape parts),

$x$ is the displacement,

$l$ is the middle length of magnetic flux lines, and $\mu_{\mathrm{r}}$ is the relative permeability of the mild steel used.

As the inductance is a function of the colocalized displacement, the displacement can be deduced. Thus, the alternative tension $\left(U_{d x}\right)$ measured has amplitude proportional to the displacement $(x)$ :

$$
U_{d x}(j \Omega)=\frac{2 E(j \Omega) x}{2 e_{0}+\frac{l}{\mu_{\mathrm{r}}}}
$$

The sensitivity depends on the iron core permeability $\mu_{\mathrm{r}}$. This characteristic grows with frequency and can vary with the temperature. Its value is based on manufacturer specifications and generally is not known with great accuracy. In a previous study [13], its value was determined experimentally and it was set to be 750 for nil frequency. Hence, it is expected that the reluctance of iron core is superior at those of air gap and the sensor sensitivity will be poor.

Finally, a demodulator is used to obtain a tension proportional to the air gap displacement value.

The effect of eddy currents is assessed to choose the bridge frequency. When the frequency of the carrier wave increases up to value greater than $10 \mathrm{kHz}$ and up to $100 \mathrm{kHz}$ the sensitivity of the IS decreases progressively.

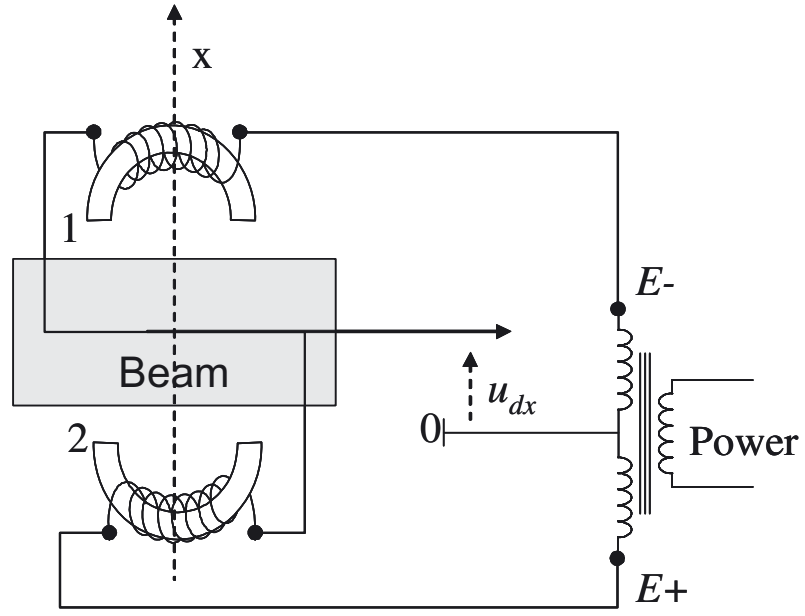

Fig. 3. Sensor circuit.

For a frequency of $200 \mathrm{kHz}$ the sensitivity of the sensor becomes almost nil [14]. Hence, the frequency of the carrier wave must be chosen as low as possible, but since the carrier wave must be suppress with a low pass filter after demodulation, it must be high enough as a function of the operational frequency range. Several tests were performed in order to set the measurement parameters. In our case a carrier wave of $10 \mathrm{kHz}$ enables a sufficient frequency bandwidth $(0-1 \mathrm{kHz})$. The amplitude of the carrier wave was chosen such to have a significant value and not to exceed the circuit capacity.

Also, and as shown by equation (2), the IS is expected to have good linearity properties over the total range of studied displacement.

\section{Preliminary numerical investigations}

This step is necessary in order to adjust and to establish reference results to be compared to those stemming from the sensor. Numerical simulations are done in a configuration as similar as possible to experiments. The beam is represented by 25 Timoshenko beam elements, taking into account the shear effect, with two nodes and 4 degrees of freedom; two displacements and two rotations per node along $x$ and $z$ directions. The spring is modeled by a supplementary stiffness element and the $(I)$ shape parts of the sensor are modeled by supplementary mass.

The structure has four bending modes in the studied frequency range. The damping effects are essentially induced by the structure. The damping was introduced by using the modal damping factor. Its value was adjusted according to experimental observations. Even if there were slight variations of their values for the different modes, a mean value of 0.003 was used for all modes (Tab. 1).

In this study, the reference distance is rebuilt by using a passage matrix $[H]$ stemming from a cubic interpolation of the modes considered for the structure:

$$
[H]=g\left(y_{i}, y_{\mathrm{a}},[\varphi]\right)
$$



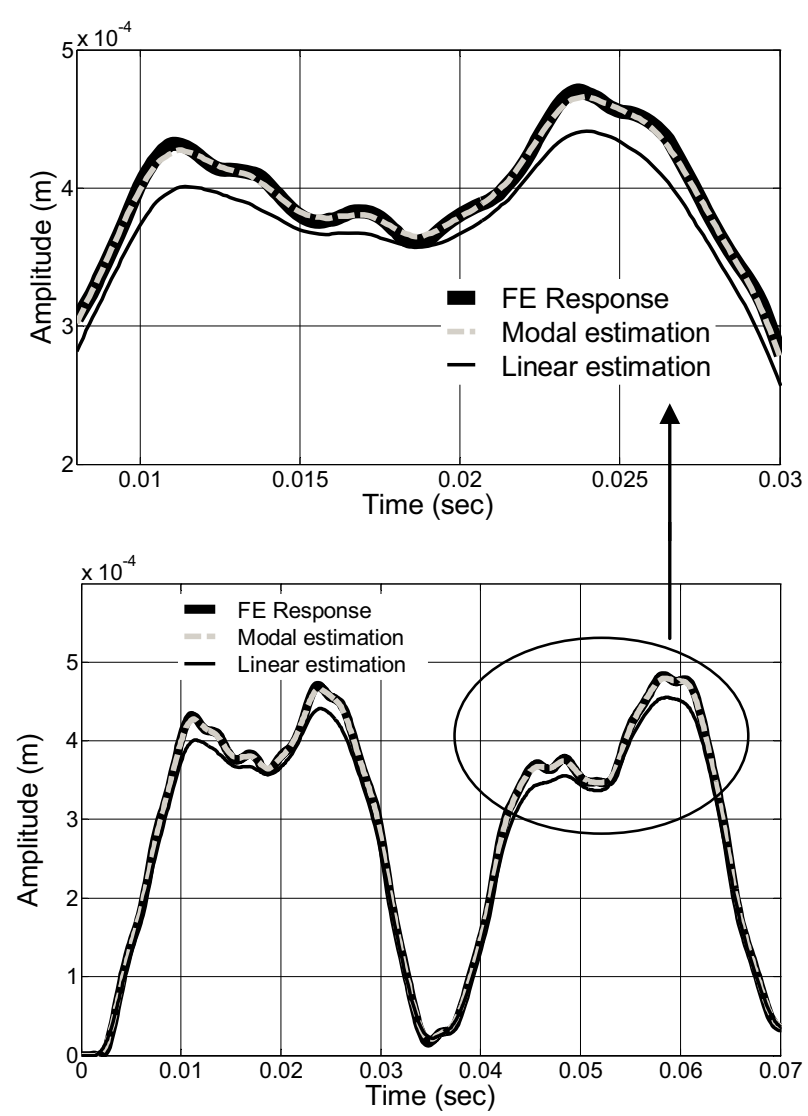

Fig. 4. Modal and linear estimation of the colocalized displacement.

Table 1. Characteristics of the model.

\begin{tabular}{ccc}
\hline Mode & Frequency $(\mathrm{Hz})$ & Damping factor \\
\hline 1 & 28.15 & 0.003 \\
2 & 58.57 & 0.003 \\
3 & 155.81 & 0.003 \\
4 & 317.02 & 0.003 \\
\hline
\end{tabular}

where $y_{\mathrm{i}}$ is the position of the $i$ th sensor, $y_{a}$ is the actuator position and $[\varphi]$ is the mode shape matrix. The modes were real, and were normalized by setting the greatest value equal to one. As we use four displacement sensors, only the four first modes are considered. The reference distance $\delta_{\mathrm{a}}$ (colocalized displacement) is then calculated in function of the measured displacements stemming for the displacement sensors $\{\delta\}$ as:

$$
\delta_{\mathrm{a}}=[\varphi]^{-1}\{\delta\}[H]
$$

In Figure 4 the reference distance calculated by using the finite element model is compared with that rebuilt either by modal estimation as described previously, or by linear interpolation of the displacements calculated at nodes \#9 and \#14, which correspond to the positions of sensors C2 and C3 placed on each side of the EMA. The accuracy of the modal estimation is acceptable and the latter is the value used for the experimental comparison.
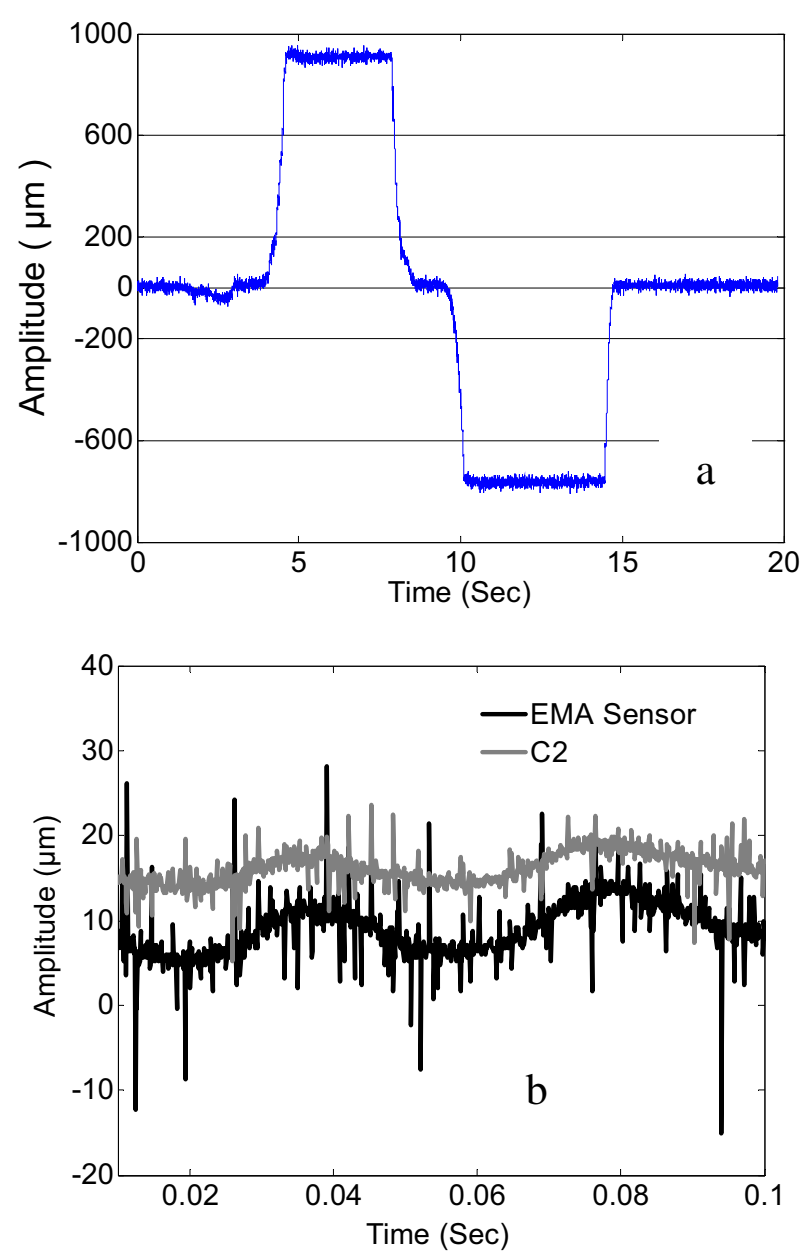

Fig. 5. Sensor calibration according to the air gap.

\section{Experimental investigations}

The IS was calibrated according to the air gap. This air gap was measured first by using classical fillers and its total value was $1.65 \mathrm{~mm}$. Then, the beam was pushed until the contact with each stator. The delivered tension was measured and the sensitivity was set to be $1.3 \mu \mathrm{m} . \mathrm{mV}^{-1}$ (Fig. 5a). In addition, when comparing the signal of the displacement sensor $\mathrm{C} 2$ to that issued form the inductive sensor, it could be noticed that the signal to noise ratio of the inductive sensor is very similar to a commercial eddy current sensors (Fig. 5b).

Impact testing was performed; several impacts were applied at different positions. The impacts were done by using the impact hammer. The impact forces measured by using the piezoelectric force sensor and the displacements by using the IS and the eddy current sensors. The signal acquisition and data processing were done by using Dspace cards, as described in the following: a calculation card (DS1005) equipped with a digital signal processor (DSP TMS320C40), a 12 bit acquisition card (DS2002) with analog to digital time conversion rate of $3.3 \mu$ s per channel, a 12 bit restitution card (DS2101) with digital to analog time conversion rate of $3.3 \mu$ s per channel. 

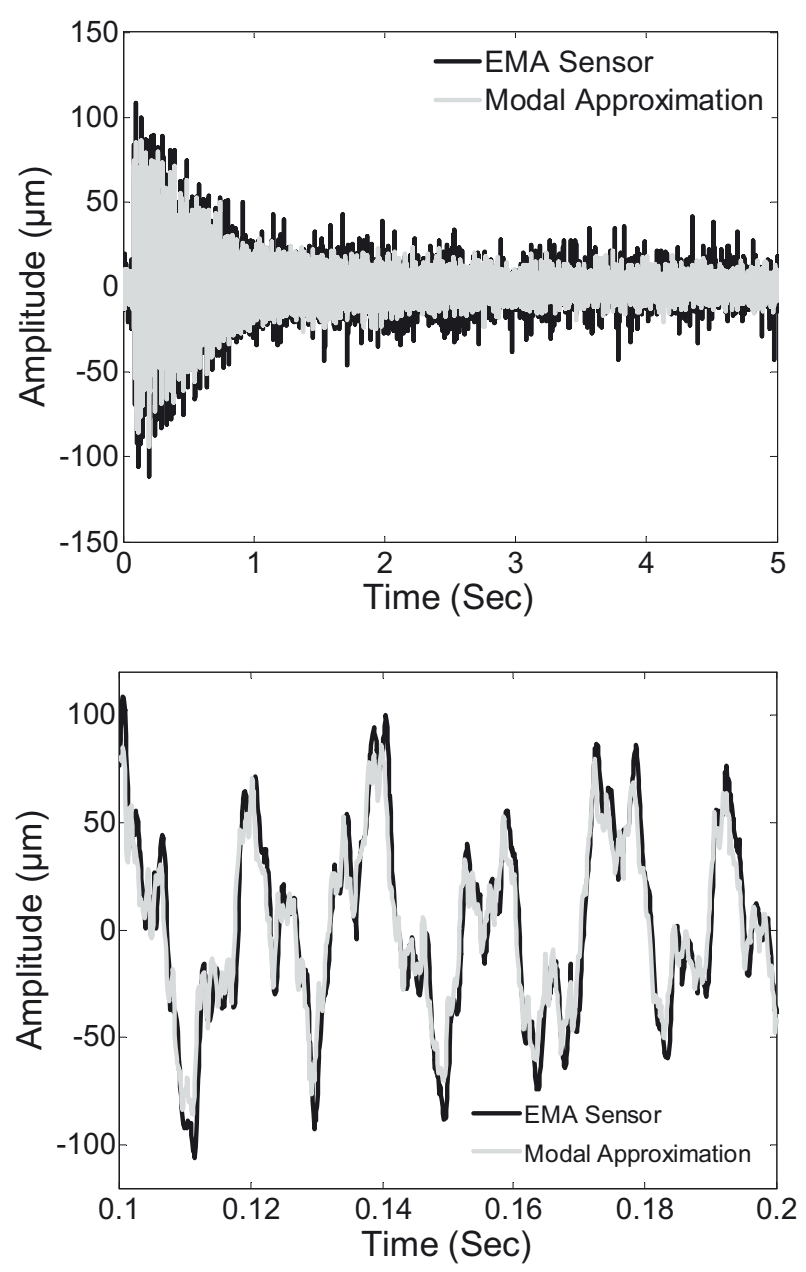

Fig. 6. Impulse response, time domain.

The displacements stemming from the eddy current sensors were recorded; were used in order to estimate, by modal approximation, the colocalized displacement. The mode shape matrix issued from the numerical calculations was utilized. In this study the first four modes were only considered. The results obtained in time domain (Fig. 6) show a good consistency.

Measurements were done for several impact locations that were selected depending on the mode shapes of the first four modes. In this study, only the results stemming from impacts performed at $0.11 \mathrm{~m}$ from the clamping end are presented. Same trends were observed for the different impact locations.

Same behavior was observed when processing the signal in frequency domain (Fig. 7). The presented frequency response was calculated as the mean value of ten time signals of $0.1 \mathrm{~s}$ without overlap. Hanning window and zero padding were used. The frequency analysis enabled to verify that the dynamic of the four first modes was well caught without gain reduction for higher mode. Therefore, the IS has a frequency bandwidth superior to the frequency range studied. It is worth mentioning that even if modal approximation was performed by using the first four modes, same trends were noticed for higher modes.

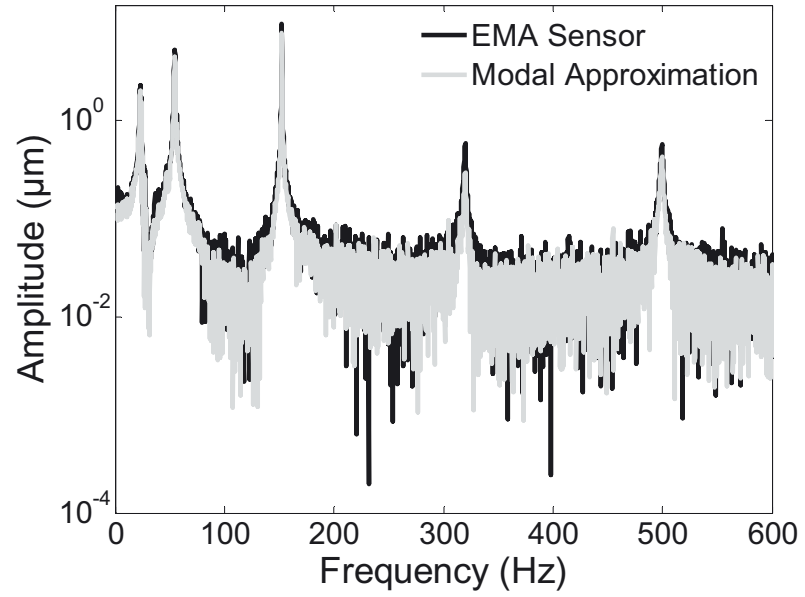

Fig. 7. Impulse response, frequency domain.

The results obtained were repetitive for different impulse testing.

\section{Conclusion}

This study is a contribution aiming at the experimental assessment of an electromagnetic actuator to behaving as inductive sensor. The electronic device was adapted in order to insert the electromagnets in an alternative Wheatstone bridge that allows the measurement of the electromagnet inductance and consequently the displacement variation. This modification is simple and could be easily implemented in industrial applications.

The results obtained demonstrate the effectiveness of the EMA behaving as a sensor. The signal to noise ratio is equivalent to a commercial eddy current sensors and IS exhibits a sufficient linearity over the utilization frequency range. The obtained measurements were repetitive. The results show that the same electromagnet configuration is suitable to actuate and to sense. In addition, the two signals have frequency bandwidth extremely different which means that self-sensing depends on the technical possibility of providing power and the measurement signal in the same device.

Modal approximation of the colocalized displacement was performed and compared to the measured one by using the IS. Good agreement was observed. This is an encouraging result toward the development of methodology using a minimum number of sensors.

\section{References}

[1] G. Schweitzer, H. Bleuler, A. Traxler, Active Magnetic Bearings - Basics, Properties and Applications, vdf Hochschulverlag AG, ETH, Zurich, 2003

[2] E.H. Maslen, Smart Machine Advances in Rotating Machinery, Proceeding of the 9th International Conference on Vibrations in Rotating Machinery, IMechE, Exeter, UK, 2008, pp. 3-14 
[3] G. Schweitzer, E.H. Maslen, Magnetic Bearings, Theory, Design, and Application to Rotating Machinery, Springer-Verlag, 2009

[4] D. Vischer, H. Bleuler, A new approach to sensorless and voltage controlled AMBs based on network theory concepts, Proceedings of the 2nd ISMB, 1990

[5] E.H. Maslen, Self-sensing for active magnetic bearings: overview and status, Proceedings of the 12th ISMB, 2010

[6] L. Kucera, Robustness of self-sensing magnetic bearing, Proceedings of MAG'97 Industrial Conference and Exhibition on Magnetic Bearings, 1997

[7] N.M. Thibeault, R.S. Smith, Magnetic bearing measurement configurations and associated robustness and performance limitations, ASME J. Dyn. Syst. Meas. Control 124-4 (2002) 589-598

[8] M.D. Noh, E.H. Maslen, Self Sensing Active Magnetic Bearings Based on Parameter Estimation, IEEE Trans. Instrum. Meas. 46-1 (1997) 45-50
[9] T. Mizuno, K. Araki, H. Bleuler, Stability analysis of self-sensing magnetic bearing controllers, IEEE Trans. Control Systems Technol. 4-5 (1996) 572-579

[10] M. Brunet, B. Wagner, Innovation: Self-sensing technology, simplified mechanical design, In S2M News (2005)

[11] H. Habermann, Paliers magnétiques, Techniques de l'ingénieur, traité Génie mécanique B 5 345, 1984

[12] J. Mahfoud, J. Der Hagopian, Fuzzy Active Control Of Flexible Structures By Using Electromagnetic Actuators, ASCE's Journal of Aerospace Engineering 24 (2011) 329337

[13] J. Der Hagopian, J. Mahfoud, Electromagnetic Actuator Design for the Control of Light Structures, Smart Structures and Systems 6 (2010) 29-38

[14] J.-L. Charron, Mesures sans contact, Techniques de l'ingénieur, Mesures Analyses R1331, 2003 